\title{
Jumping of Flocks in the Electric Field in the Process of Electro-Static Flocking
}

\author{
By Yoshiharu Mukai, Member, TMSJ \\ Textile Research Institute of Osaka Prefecture, Osaka.
}

\begin{abstract}
The jumping of nylon flocks in the electric field has been investigated quantitatively with a photo density meter, a leakage resistance meter.

The results are:

1) The flying ability of flocks in the electric field under a given electric stress begins to increase at a leakage resistance of about $10^{11}$ and continues to rise rapidly as the leakage resistance decreases. However, the jumping ability reaches a saturation point when the leakage resistance is about $10^{\circ}$.

2) The flying density of flocks changes in proportion to the separability of fibers if the jumping ability is the same.
\end{abstract}

\section{Introdution}

The jumping of flocks in the electric field is one of the fundamental and most important of electro-static flocking.[1] Yet, little has been done to clarify jumping. Some experts have dealt with the fundamental principles of flocking by electro statics. In actual electro-static flocking, however, the principles are often confused by other elements.

Rapid advance of adhesives by high polymer chemistry in recent years has opened a new field of application for flocking. Also materials for flocks have changed from waste wool and rayon to synthetic fibers.

Therefore, the importance has been recognized anew of analyzing and explaning the jumping condition of flocks in the electric field, for the purpose of using synthetic fibers of low moisture absorption, which, without treatment, do not make electro static jumping easy.

The author has endeavored to clarify the socalled jumping treatment for establishing the industrial method of synthetic fiber-flocking, which is at present difficult, by inquiring quantitatively into the various conditions for flocking. For this purpose, a photo density meter newly devised and a leakage resistance meter have been used.

\section{Experiments}

It has been commonly knowledge that the di- electric charge of a material in the electro-static field changes by the electric stress $(V / M)$, the dielectric constants of both the material and the atomosphere.[2] In addition, there has been much inquiry made into electric charge by the mechanical friction, in quest of the so-called anti-charge.[3] However, it is difficult, in practice, to measure the dielectric constant of flocks related to the jumping of flocks in the actual flocking process.

It is not enough to investigate static nature of flocks alone. The important thing is to clarify the relation of the electro static natures of flocks to their jumping ability.

The dielectric constant of a solid changes by moisture absortion, and influences the jumping of flocks.

Therefore, the author tried, first, a treatment aimed to increase the moisture absorption of synthetic fibers. However, it had little influence on the flying density of flocks treated, irrespective of the treating agents used, as shown by Fig. 1.

Accordingly, the author divided the factors for flying of flocks into two. One is the jumping ability which changes by the electric condition, the other being the separability of fibers.

The anti-charge ability and the moisture absorption tend to increase the jumping ability of flocks but lessen their separability.

To improve the jumping ability, we used inorganic salts or anti-charge agents, or both. To improve separability, we used soft-finish agents, that is, slip agents. 


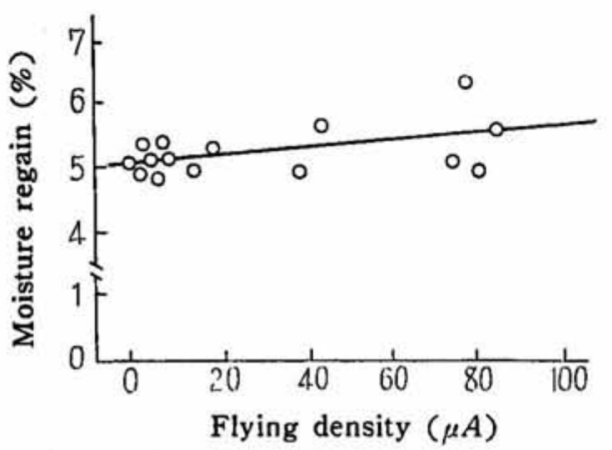

a) Flocks: Nylon $13 \mathrm{~d}, 3 \mathrm{~mm}$ cut.

b) Threated with $5 \%$ solution of each agent. Bath ratio $10: 1$; dipped for 30 mins. at $40^{\circ} \mathrm{C}$, hydrated with $25 \%$, pick-up.

c) Measured at $16^{\circ} \mathrm{C}, 75 \%$ R.H. after nature-dried for $48 \mathrm{hrs}$. after treatment.

Fig. 1 Moisture regain and flying density of treated flocks

\section{2-1. Flocks experimented}

The flocks were composed of $13 \mathrm{~d}$ nylon, $3 \mathrm{~mm}$ cut. They were dipped in $1 \%$ pure-water solution of the agent shown in Table 1 , or in a $2 \%$ combined solution of two agents dissolved in equal proportions.

The flocks were dipped for 10 minutes at $40^{\circ} \mathrm{C}$, with the bath ratio of $20: 1$. They were then dehydrated to the extent of about $100 \%$ pick-up, spread properly and air-dried in the room.

\section{2-2. Method of measurement and apparatus}

\section{a) Leakage resistance}

The leakage resistance of flocks was measured with two measuring taps (injection needles) planted on the tip of the glove of Type II static-scope, made by Oki Electric Industry Co. (see Fig. 2), at each flying density.

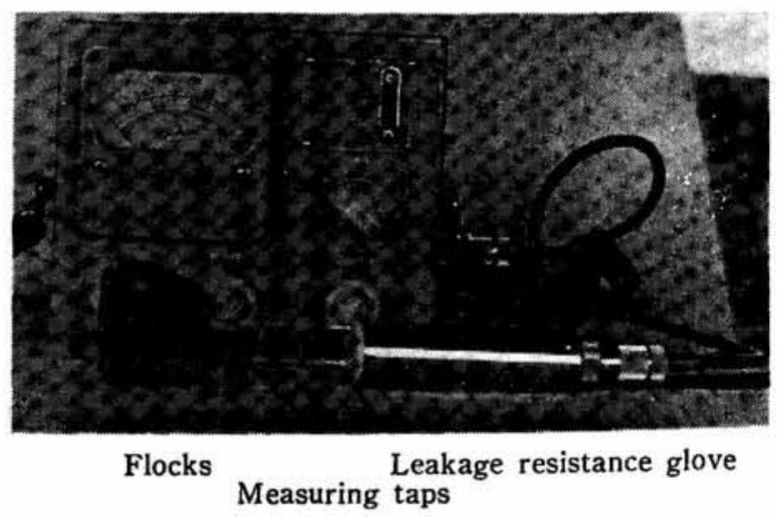

Fig. 2 Static-scope

\section{b) Separability}

A sieving apparatus with a 550-stroke pulsation per minute was used. Separability was determined from the volume of flocks passing through the gauze of the sieving apparatus.

\section{c) Flying density}

An electro-static field was built between the light source and the photo receiving part of the photo density meter, as shown in Fig. 3. The interception of light bundles incident to the jumping of flocks immediately after the closing of secondary circuit of a D.C. high-voltage generator was determined with the percentage differential current meter.

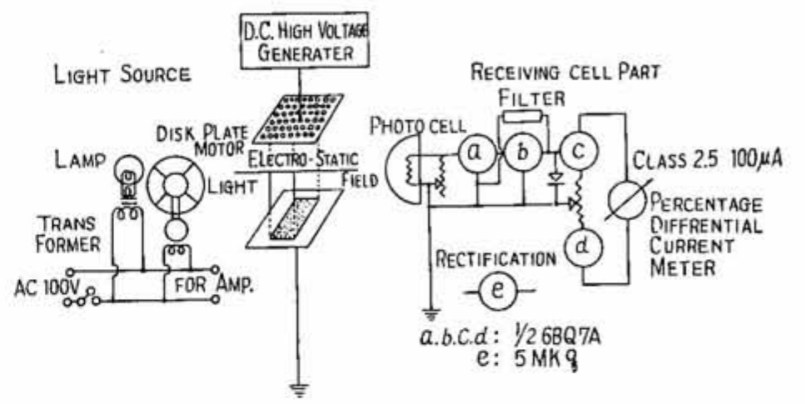

Fig. 3 Photo density meter and electro-static field

Two devices were installed to prevent external disorders. One was an automatic voltage stabilizer in the primary circuit, the other being a disk plate (with slits) revolving at a fixed speed along the light axis. This made the cast light bundle an intermittent one of about 200 cycles. The output of the photo-cell was amplified to know the difference in jumping abilities of various flocks.

ii) Electro-static field

An earth-connected pole (aluminium plate) was horizontally placed on a chasis built of hard polyvinyl chloride, the position of the pole being 850 millimeters above the floor surface. A high-voltage pole (multi-hole aluminium plate) was placed $90 \mathrm{~mm}$ above the earth-connected pole. Flocks were spread over the fixed position having a $250 \times 100 \mathrm{~mm}$ area of the earth-connected pole plate. The amount of flocks supplied for each exeriment was $1 \mathrm{~g}$ (4.0 $\mathrm{mg} / \mathrm{cm}^{2}$ ).

iii) Generator

A D.C. high-voltage generator made by the Japan Electro-Static Flocking Industry Co. was used. Its capacity 0.25 K.V.A., the secondary output being D.C. 15 K.V. (Effective values).

$$
\text { gocornal of The 7extile Machinery Society of Dapan }
$$




\section{Results of Experiments and Discussion}

3-1. Variations in the flying density due to various leakage resistance

We prepared several groups of flocks having various degrees of leakage resistance by varying the moisture absorption and the concentration of dissolved agents; the agents selected were those which would promote the separability and jumping ability of flocks. Out of such groups of flocks we picked those which were almost equal in separability. The variations in the flying density due to differences in the degree of leakage resistance was then investigated with the results given in Fig. 4. The figure shows that, if we ignore the slight difference in separability, the flying density is comparable to the jumping ability of flocks.

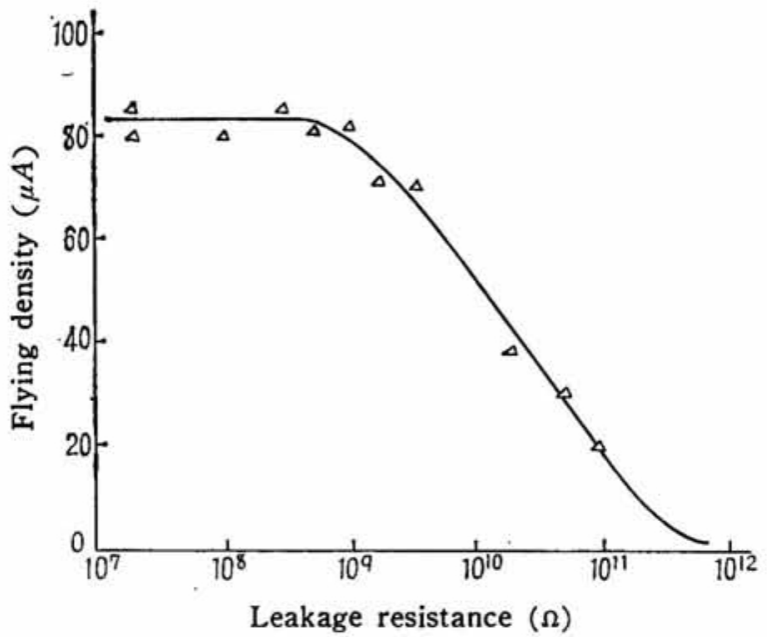

Fig. 4 Flying density and leakage resistance

Therefore, the jumping ability of flocks in the electro-static field under a given electro stress changes by the function of the logarythm of leakage resistance of flocks, if we ignore the difference in separability between flocks and the dielectric constant of atomosphere.

The jumping ability of flocks begins to increase at a leakage resistance of about $10^{11} \Omega$, and continues to rise rapidly as the leakage resistance decreases, reaching a saturation point when the leakage resistance is about $10^{9} \Omega$. However, the jumping ability below a certain limit is meaningless in practical use if we consider the planted strength, the planting density, etc.

The jumping ability reaches the maximum when the leakage resistance declines to about $10^{\circ} \Omega$.
Accordingly, leakage resistance need not be lowered too far during the jumping treatment.

3-2. Variations in the flying density due to the difference in separability between flocks

The separability and flying density of flocks of comparatively low leakage resistance and dried for 40 hours in the room were measured with the results shown in Table 1.

Table 1 Leakage resistance, separability and flying density of flocks

\begin{tabular}{|c|c|c|c|c|c|c|}
\hline \multirow{2}{*}{$\begin{array}{l}\text { Ma- } \\
\text { terial }\end{array}$} & \multicolumn{3}{|c|}{ Agent } & \multicolumn{2}{|c|}{$\begin{array}{l}\text { LeakageSepa- } \\
\text { re- rability }\end{array}$} & \multirow{2}{*}{$\begin{array}{l}\text { Fly- } \\
\text { ing } \\
\text { densit } \\
(\mu A)\end{array}$} \\
\hline & Mark & Class & $\begin{array}{l}\text { Chemical } \\
\text { composition }\end{array}$ & $\begin{array}{l}\text { sistance } \\
(\Omega)\end{array}$ & $(\%)$ & \\
\hline $\mathrm{F}_{1}$ & A & \multirow{2}{*}{ Inorganic salt } & Table salt & $2 \times 10^{7}$ & 53.3 & 70 \\
\hline $\mathrm{F}^{2}$ & B & & Silicate & $5 \times 10^{9}$ & 67.3 & 70 \\
\hline$F^{3}$ & \multirow{4}{*}{\multicolumn{2}{|c|}{$\begin{array}{l}\text { Parmanent } \\
\text { J) anti-charge } \\
\text { agent } \\
\text { K) }\end{array}$}} & \multirow{4}{*}{$\begin{array}{l}\text { Cationic } \\
\text { water } \\
\text { solution } \\
\text { resin }\end{array}$} & $3 \times 10^{9}$ & 9.5 & 20 \\
\hline $\mathrm{F}_{4}$ & & & & $1.5 \times 10^{9}$ & 25.0 & 20 \\
\hline$F_{5}$ & & & & $4 \times 10^{8}$ & 10.0 & 20 \\
\hline $\mathrm{F}_{8}$ & & & & $4 \times 10^{9}$ & 39.5 & 60 \\
\hline$F_{7}$ & $\mathbf{E}$ & \multirow{2}{*}{$\begin{array}{l}\text { Anti-charge } \\
\text { agent }\end{array}$} & \multirow{2}{*}{$\begin{array}{l}\text { Oil agent } \\
\text { phoshoric } \\
\text { acid ester } \\
\text { system anionic } \\
\text { type }\end{array}$} & $1.5 \times 10^{8}$ & 4.5 & 20 \\
\hline$F_{8}$ & F & & & ic $4 \times 10^{10}$ & 0.5 & 0 \\
\hline$F_{0}$ & \multirow{3}{*}{\multicolumn{2}{|c|}{$\begin{array}{l}\text { Soft finish } \\
\text { agent }\end{array}$}} & \multirow{3}{*}{$\begin{array}{l}\text { Cationic } \\
\text { surface active } \\
\text { agent } \\
\text { quarternary } \\
\text { ammonium } \\
\text { salt type }\end{array}$} & $1.5 \times 10^{10}$ & 8.5 & 10 \\
\hline$F_{10}$ & & & & $4 \times 10^{9}$ & 25.0 & 30 \\
\hline$F_{11}$ & I & & & $1.5 \times 10^{8}$ & 63.2 & 80 \\
\hline$F_{12}$ & \multicolumn{3}{|c|}{$\begin{array}{l}\mathrm{C}+\mathrm{H} \\
\quad \text { Anti-charge agent } \\
\text { and soft finish agent }\end{array}$} & $3 \times 10^{9}$ & 12.5 & 25 \\
\hline$F_{18}$ & \multicolumn{3}{|c|}{$\mathrm{F}+\mathrm{K}$} & $3 \times 10^{13}$ & 3.5 & 0 \\
\hline
\end{tabular}

$\mathrm{F}_{4}, \mathrm{~F}_{5}$ : Retreated with anionic active agent (J. or $\mathrm{K}$ ), after treatment with C. Conditions for both treatments same.

$F_{12}, F_{18}$ : Treated with $1 \%$ solution each of 2 agents (2\% solution in total).

From the results described in section $3-1$, it appears that the jumping ability of flocks is nearly the same if their leakage resistance is $10^{\circ}$ or below. Accordingly, we carried on the experiment with such groups of flocks below $10^{9}$ in leakage resistanceflocks of nearly equal jumping ability.

The results are given in Fig. 5. They show clearly that the flying density of flocks changes in proportion to separability, if they are equal in jumping ability. 


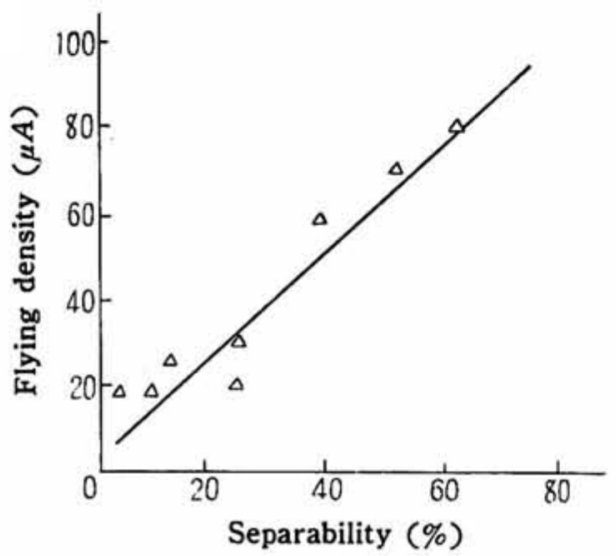

Fig. 5 Flying density and separability

The results described in 3-1. and 3-2. show, then, that the leakage resistance of $10^{9}$ or below, plus the best separability, makes jumping in the electro-static flocking most effective.

3-3. Variations in the flying density due to the moisture absorption of flocks

The wetness of flocks under actual weather conditions changes every moment with fluctuations in the temperature and humidity. Therefore, the leakage resistance and the separability vary with each kind of treating agent. Thus, the flying density also changes sharply with fluctuations in the temperature and humidity.

Flocks of comparatively good separabilty were measured with the changes in the flying density induced by fluctuations in their leakage resistance and in weather conditions. The results are given in Fig. 6.

The abcissa in Fig. 6 show the time after the jumping treatment, followed by 40 -hour natural drying and one-hour drying in hot air at $70^{\circ} \mathrm{C}$.

The figure shows that the flying density begins to increase with an increase in the moisture absorption of flocks, i.e., with a decrease in their leakage resistance. It approachs the peak the next morning. Then it decreases with an increase in the leakage resistance induced by drying. After two days, it reaches the peak to keep balance with high humidity. Thereafter, only flock $F_{1}$ decreases abruptly in flying density because its separability drops close to 0 .

Differences in flying density arose between groups of flocks having equal leakage resistance. This was due to the differences in separability between flocks.

$F_{2}$ had a comparatively good flying density despite its comparatively high leakage resistance.
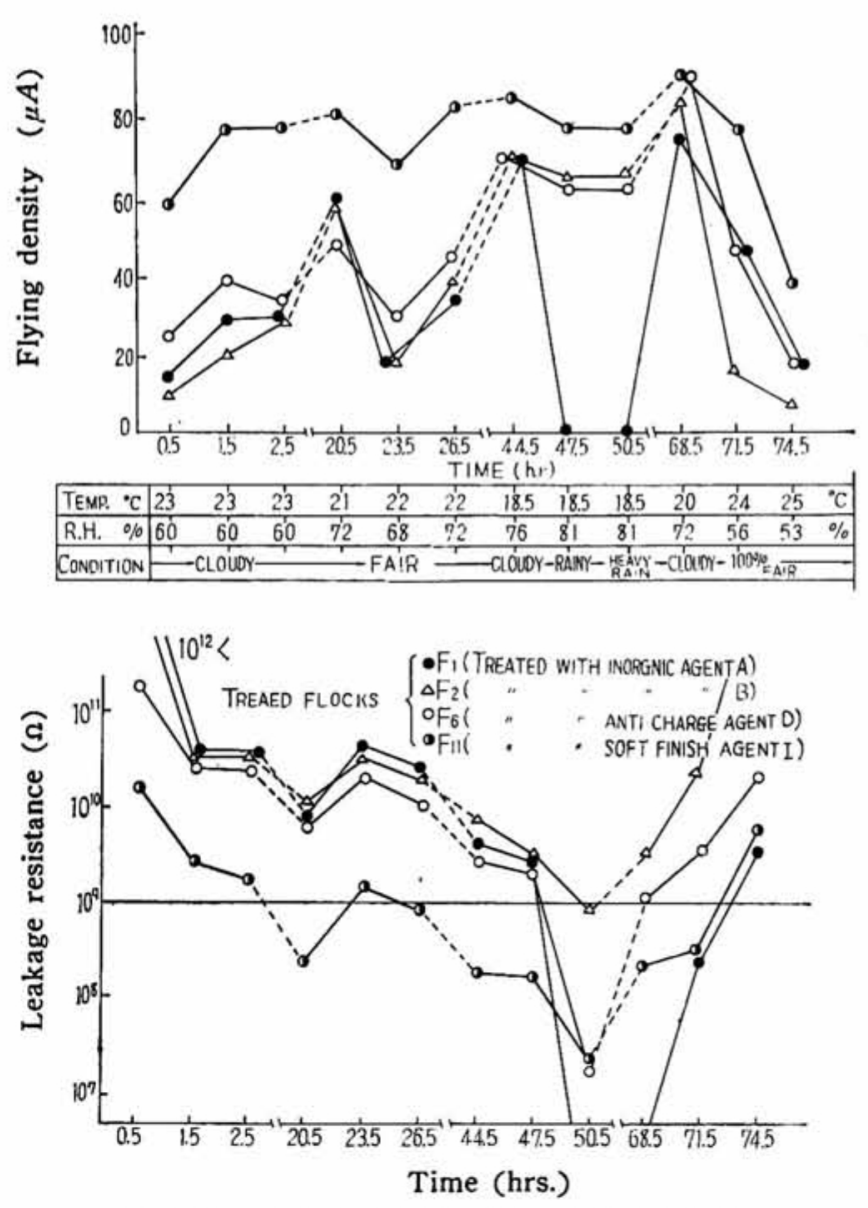

Fig. 6 Flying density and weather condition

This is because $F_{2}$ had exceptionally good separability, which decreased only slightly despite the high humidity.

This means that an increase in the moisture absorption of flocks lowers their leakage resistance and raises their jumping ability. However, wetness reduces separability and impairs the jumping ability. Generally, the leakage resistance absorption decreases by desorption. Accordingly, flock treatment should be such that it increase leakage resistance slightly during drying, and reduces separability sligtly when wet.

Of all treating obtainable, a cationic surface active agent of quaternary ammoniun salt type (I) has been found highly effective for the jumping treatment of flocking, as shown with $F_{11}$ in Fig. 6 . Inorganic salt (B) (soliate) is one of the agents widely used today, but a flock treated with this agent, although it decreases only slightly in separability when wet, increases sharply in leakage resistance during drying, thus reducing the jumping ability abruptly, as shown by $F_{2}$ in Fig. 6 . 
These studies have led us to clarify the causes of confusion so often encountered in the flocking job. One remedy is to use a treating agent like I. Another conceivable remedy is to suit the temperature and humidity to the treating agent used (e.g., about $20^{\circ} \mathrm{C}$ and $75 \%$ R.H. for $B$ ), enforcing strict control of air condition (especially humidity) in not only the flocking chamber but in the stock chamber, too, keeping a well-balanced humidity in both chambers.

\section{Conclusions}

The condition for jumping of flocks in the electric field has been clarified quantitatively. This was aided with a photo-density meter trially devised, and a leakage resistance meter.

It has been found that only a small arnount of a cationic surface active agent of the quaternary ammonium salt type is highly effective for the jumping treatment of synthetic-fiber flocking.

It is believed that inquiry into new treating agents, such as permanent anti-charge agents and soft finish agents, will be interesting and useful, because these new agents have many good properties, compared with the usual inorganic salts, for the handling of after-flocking. They have also tenacity and pliability, such as are found in acrylic adhesives.

\section{Literature cited}

[1] (Inter alia) S. Akiyama, M. Tanaka and Y. Mukai; J. Soc. Text. Cell. Ind., 17, p. 60, (1961-1)

[2] T. Kinoshita; High Voltage Condition, OHM Sha, Co., Tokyo. p. 21 (1955)

[3] (Inter alia) K. Sasaki and K. Ban; J. Soc. Text. Cell. Ind., 17, p. 458 (1961-5) 